\title{
Auditory Alert System for Fluorescence-Guided Resection of Gliomas
}

\author{
-Technical Note-
}

\author{
Satoshi UtSUKI, Hidehiro OKA, Yoshiteru MiYaJIMA, Satoru SHIMIZU, \\ Sachio SUZUKI, and Kiyotaka FUJII
}

Department of Neurosurgery, Kitasato University School of Medicine, Sagamihara, Kanagawa

\begin{abstract}
Protoporphyrin IX (PPIX) fluorescence-guided brain tumor resection using 5-aminolevulinic acid labeling is one of the most valuable tools available to determine the extent of glioma infiltration, but requires repeated spectroscopic evaluation of the tissue. The present method informs the surgeon of residual tumor in real time using spectrum analysis of PPIX fluorescence and an audible alert system. The target region was illuminated with a laser with a peak wavelength of $405 \pm 1 \mathrm{~nm}$ in addition to the usual microscope halogen lamp during tumor resection. Analysis of the spectrum detected the PPIX peak using a difference in relative intensity exceeding 500 at $636 \mathrm{~nm}$ and $632 \mathrm{~nm}$, when an audible alert was transmitted to the surgeon. Using this method, infiltration of glioma was detected and confirmed histologically in three of six glioblastomas. The surgeon can detect tumor infiltration far more objectively and with less effort using this system during tumor resection.
\end{abstract}

Key words: 5-aminolevulinic acid, auditory alart, fluorescence-guided resection, protoporphyrin IX

\section{Introduction}

Glioblastoma must be removed as soon as possible after detection by surgery. ${ }^{1)}$ Protoporphyrin IX (PPIX) fluorescence-guided brain tumor resection using 5-aminolevulinic acid (5-ALA) labeling is one of the most useful methods to determine the extent and guide the removal of infiltrating glioma, but depends upon the subjective judgment of the operator. ${ }^{2,3)}$ Infiltration of tumor cells into the non-fluorescent area is known to occur, because the tumor has recurred even after removal under PPIX fluorescence guidance. ${ }^{4)}$ Standardized methods of PPIX fluorescence-guided tumor resection are desirable to eliminate subjective judgments. One method is to monitor for the presence of the PPIX spectrum. ${ }^{6}$ Spectroscopy detection of PPIX fluorescence provides a far more objective and sensitive measure of the infiltration of the tumor than the naked eye.6) However, the spectral analysis of PPIX is displayed on a computer screen and is usually relayed to the surgeon via an assistant during tumor resection.

We describe a simple, useful, and accurate audible

Received May 22, 2007; Accepted November 6, 2007 alert system for direct feedback of PPIX fluorescence to the surgeon during fluorescence-guided resection.

\section{Technique}

This study was reviewed and approved by the Kitasato University Hospital Ethics Committee, and informed consent was obtained from the patients. Two hours before administration of anesthesia, the patient was given a 1-g oral dose of 5-ALA. After resection of the tumor bulk, the limited target region was illuminated with a semiconductor laser (VLDV1 version 2; $M$ \& M Co., Ltd., Tokyo) with a peak wavelength of $405 \pm 1 \mathrm{~nm}$ and an output of $40 \mathrm{~mW}$ directed at the tumor locus by the surgeon using a hand-held, fiber-optic cable. The tissue infiltrated by the tumor was removed under PPIX fluorescence guidance using the naked eye, then our new audible alert system.

The audible alert system was based on a spectrometer and accessory software (BW-Spec V3.09; B \& W TEK, Inc., Newark, Del., U.S.A.) to analyze the spectrum waveform. The PPIX fluorescence spectrum has two peaks, a sharp peak at $636 \mathrm{~nm}$ and a slightly broader peak at $705 \mathrm{~nm}$. PPIX fluorescence is impos- 


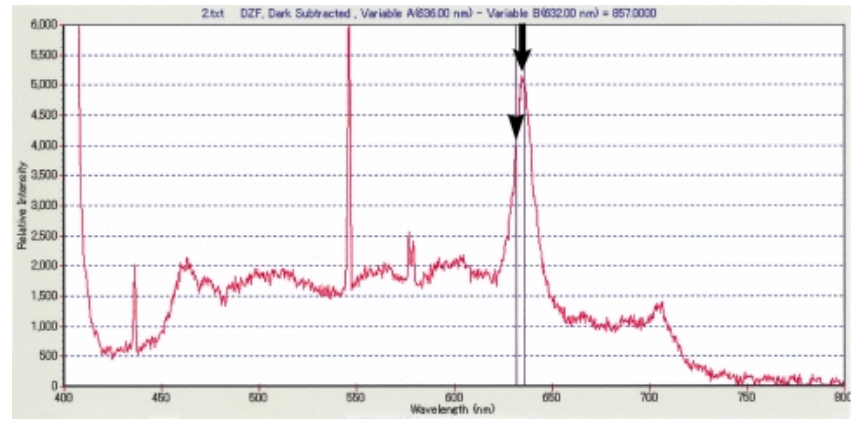

Fig. 1 Spectrum analysis using accessory software (BW-Spec V3.09). Spectral intensity is increased between $400 \mathrm{~nm}$ and $700 \mathrm{~nm}$ in wide areas due to exposure to a microscope halogen lamp. However, the two peaks of $636 \mathrm{~nm}$ and $705 \mathrm{~nm}$ indicating protoporphyrin IX are observed. The steep rise in intensity at the peak of $636 \mathrm{~nm}$ was used to calculate the difference in intensity at $636 \mathrm{~nm}$ (arrow) and $632 \mathrm{~nm}$ (arrowhead) of 857 in this case.

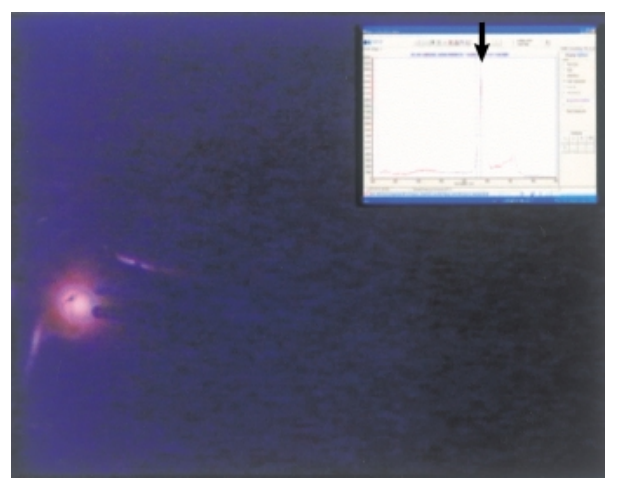

Fig. 2 Monitor screen of the microscope field showing red fluorescence of protoporphyrin IX, and the real time spectrum (top right insert) showing a peak at $636 \mathrm{~nm}$ (arrow).

sible to distinguish from fluorescent lamp or microscope halogen lamp illumination because these lamps provide a broad light spectrum that includes $636 \mathrm{~nm}$. Therefore, we developed a method to specifically detect the steep peak of PPIX even in the presence of a broad ambient light spectrum. The accessory software was set up to trigger an audible signal if the difference in relative intensity between 636 $\mathrm{nm}$ and $632 \mathrm{~nm}$ exceeded 500 (Fig. 1). There was no variation in the sound intensity with different relative intensities. Consequently, the audible signal indicated tissue regions with PPIX fluorescence (Fig. 2). Therefore, the surgeon could detect the presence of even weak PPIX fluorescence invisible to the naked eye (Fig. 3).

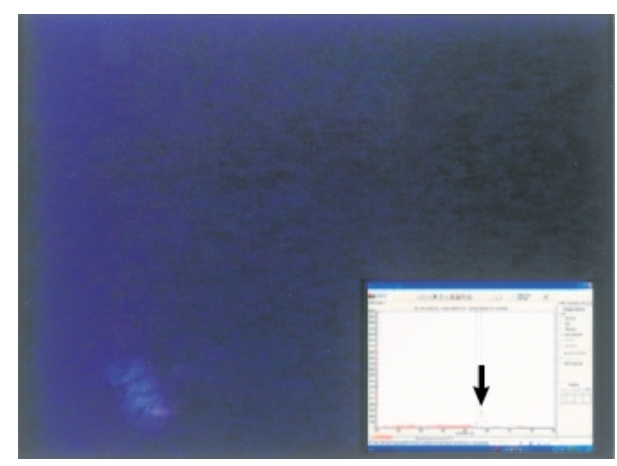

Fig. 3 Monitor screen of the microscope field showing only blue light, which is the excitation light wavelength, but no red fluorescence of protoporphyrin IX, and the real time spectrum (bottom right insert) showing a low peak at $636 \mathrm{~nm}$ (arrow).

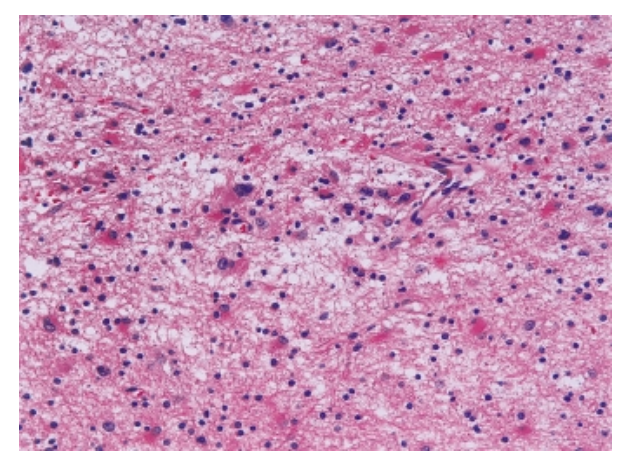

Fig. 4 Photomicrograph showing infiltration of nuclear pleomorphic tumor cells. Hematoxylin and eosin stain, original magnification $\times \mathbf{1 0 0}$.

The new method permitted additional resection of noneloquent brain tissue under the normal surgical illumination necessary to detect structures such as blood vessels. This technique was used in six patients with glioblastoma. The audible alert sounded in areas with visible PPIX fluorescence in all patients, and in areas with invisible PPIX fluorescence in three patients. Histological examination confirmed the presence of infiltrated neoplastic cells in all these areas (Fig. 4).

\section{Discussion}

With conventional methods, an assistant usually informs the surgeon of the spectral analysis results after resection and not during the process of removing the tissue. Therefore, the resected area has to be checked repeatedly. With the present method, the 
operator can assess tumor infiltration in real time based on the audible alert indicating detection of the PPIX peak, so tumor resection can be completed with less stress on the surgeon.

Surgeons try to remove all tissue with PPIX fluorescence as far as possible to eliminate any areas of glioma invasion if the area is noneloquent. All tissue with PPIX fluorescence is removed in eloquent areas under motor evoked potential or awake surgery monitoring. PPIX fluorescence may be observed even in the absence of tumor infiltration in glioma, but most cases are associated with tumor infiltration. ${ }^{5}$

\section{Acknowledgment}

This work was supported in part by Grants-in-Aid for Scientific Research from the Ministry of Education (Nos. 16790830, 19591700), and a Japan Society for the Promotion of Science Fujita Memorial Fund for Medical Research.

The authors gratefully acknowledge the technical assistance of Ms. Yoshie Yasui.

\section{References}

1) Hollerhage HG, Zumkeller M, Becker M, Dietz H: Influence of type and extent of surgery on early results and survival time in glioblastoma multiforme. Acta Neurochir (Wien) 113: 31-37, 1991

2) Kaneko S: [Intraoperative photodynamic diagnosis of human glioma using ALA induced protoporphyrin IX]. No Shinkei Geka 29: 1019-1031, 2001 (Jpn, with Eng abstract)

3) Stummer W, Novotny A, Stepp H, Goetz C, Bise K, Reulen HJ: Fluorescence-guided resection of glioblastoma multiforme by using 5-aminolevulinic acidinduced porphyrins: a prospective study in 52 consecutive patients. J Neurosurg 93: 1003-1013, 2000

4) Stummer W, Pichlmeier U, Meinel T, Wiestler OD, Zanella F, Reulen HJ; ALA-Glioma Study Group: Fluorescence-guided surgery with 5-aminolevulinic acid for resection of malignant glioma: a randomised controlled multicentre phase III trial. Lancet Oncol 7: 392-401, 2006

5) Utsuki S, Oka H, Sato S, Shimizu S, Suzuki S, Tanizaki Y, Kondo K, Miyajima Y, Fujii K: Histological examination of false positive tissue resection using 5aminolevulinic acid-induced fluorescence guidance. Neurol Med Chir (Tokyo) 47: 210-214, 2007

6) Utsuki S, Oka H, Sato S, Suzuki S, Shimizu S, Tanaka S, Fujii K: Possibility of using laser spectroscopy for the intraoperative detection of nonfluorescing brain tumors and the boundaries of brain tumor infiltrates. Technical note. J Neurosurg 104: 618-620, 2006
Address reprint requests to: Satoshi Utsuki, M.D., Department of Neurosurgery, Kitasato University School of Medicine, 1-15-1 Kitasato, Sagamihara, Kanagawa 228-8555, Japan.

e-mail: utsuki@med.kitasato-u.ac.jp

\section{Commentary}

For about 10 years, a photodynamic diagnosis has been used to identify tumor-infiltrative lesions during surgery for malignant glioma by administering 5aminolevalinic acid (ALA, a precursor of heme) and detecting the fluorescence of protoporphyrin IX (PpIX, a metabolite of ALA) emitted from the tumor cells which have incorporated the ALA. In Japan, this technique has recently been used at many institutes, and its usefulness has been demonstrated.

This study describes the method of an auditory alert system instead of visual information. The idea is indeed novel, but there is no need for use of such a system if an adequate light source is used during surgery. If the light source used is weak, as shown in Figs. 2 and 3 , it is necessary to check the spectrum on a screen. When checking the spectrum, the surgeon must shift their gaze from the operative field during surgery and, for this reason, an auditory alert system might be useful. However, if a high-power light source is employed, distinction between strong and vague fluorescence is possible simply by observation, and the surgeon can continue surgical manipulations under the wide fluorescent field while checking for fluorescence and thus complete surgery quickly. Furthermore, a high-power light source is also used in neuroendoscopy, etc. ${ }^{3)}$ Unlike the auditory alert system, which allows only distinction between positive and negative areas, appropriate observation using an adequate light source also allows comparison of the different intensity of fluorescence between different areas.

In addition, photodynamic diagnosis using ALA has several problems and questions remain. First, it is unknown why PpIX level is high in tumor cells. It is also unknown why some cases of malignant glioma emit fluorescence of PpIX while others do not. It is not possible to predict emission of PpIX fluorescence preoperatively in a given case. Furthermore, it is well known that fluorescence of PpIX is also observed in areas free of tumor cells. ${ }^{2)}$ It is also well known that fluorescence is seen in cases of benign tumors such as meningioma, ${ }^{11}$ and that fluorescence is not observed in malignant tumors such as metastatic brain tumor but is noted in the surrounding tumor-free areas. The possibility of false-positive and false-negative judgments must therefore be taken into account when the 
ALA is used. Other important points are the possibility of photobleaching (attenuation of fluorescence due to light) and change in the spectrum due to interference by external light. Change in spectrum usually depends on the angle of the light source. As a result, whether the auditory alert system can function effectively depends on the angle of the light source and various other conditions even if the same area is evaluated.

It needs to be borne in mind that use of the auditory alert system can increase the risk of surgery unless these problems and questions are fully taken into account.

\section{References}

1) Kajimoto $Y$, Kuroiwa $T$, Miyatake $S$, Ichioka $T$, Miyashita M, Tanaka H, Tsuji M: Use of 5-aminolevulinic acid in fluorescence-guided resection of meningioma with high risk of recurrence. J Neurosurg 106: 1070-1074, 2007

2) Miyatake S, Kuroiwa T, Kajimoto Y, Miyashita M, Tanaka H, Tsuji M: Fluorescence of non-neoplastic, magnetic resonance imaging-enhancing tissue by 5aminolevulinic acid: case report. Neurosurgery 61: 1101-1104, 2007

3) Tamura Y, Kuroiwa T, Kajimoto Y, Miki Y, Miyatake S, Tsuji M: Endoscopic identification and biopsy sampling of an intraventricular malignant glioma using a 5aminolevulinic acid-induced protoporphyrin IX fluorescence imaging system. J Neurosurg 106: 507-510, 2007

Toshihiko Kuroiwa, M.D. Department of Neurosurgery Osaka Medical College Takatsuki, Osaka, Japan

The authors present an interesting enhancement to the accuracy and dependability of 5ALA fluorescent detection of GBMF residual tumor during attempts at gross total resection. Spectroscopic analysis of the presence and degree of tissue fluorescence is done with a method that does not allow the normal microscope illumination to obscure the signal, and the presence of tumor cell fluorescence is indicated in a proportional fashion with an audible alert system. This clever technical adjunct has the potential to achieve more complete resections, particularly of infiltrating tumor.

Edward R. Laws, Jr., M.D., F.A.C.S., D.Med.Chir. Naples. (Hon), F.R.C.S.Ed. (Hon), F.R.C.P.S.G. (Hon)

Department of Neurosurgery

Pituitary/Neuroendocrine Center

Stanford University Medical Center

Advanced Medicine Center

Stanford, California, U.S.A.

The management of malignant gliomas continues to be an enormous challenge. We continue to make strides in terms of multi-modality management, increasing the percentage of patients who are living longer than one year. Additional techniques that maximize the benefit of initial surgical resection are increasingly valuable. In many patients, we are "already behind the eight ball" by the time the patient comes to clinical recognition. This occurs because of the many features of glioblastoma which affect our patients worldwide: heterogeneity of the tumor burden within the tumor mass itself, regional infiltration of the tumor in adjacent brain with critical function, infiltrative borders which defy our ability to safely resect even with advanced image guidance, a high likelihood of recurrence at the edge of the tumor resection, and a large tumor burden remaining even after maximal resection. Shapiro once hypothesized that a newly recognized 30-60 g glioblastoma had 3-6 $\times$ $10^{10}$ tumor cells at the time of clinical presentation. With a 90\% resection, 3-6 $\times 10^{9}$ remain, and with a $99 \%$ resection (surely this would be considered an excellent surgery), 3-6 $\times 10^{8}$ tumor cells remain. ${ }^{1)}$ Since even excellent surgery cannot cure glioblastoma, how do we manage the remaining tumor burden after maximal safe resection? Unfortunately, multi-center trials that assess the maximum safe resection of gliomas, select patients who have tumors in areas of the brain where safe resection is feasible. This feature per se selects patients that will fare better when surgery, radiation, and perhaps chemotherapy are used.

Utsuki and his colleagues report an innovative strategy to recognize tumor infiltrated brain using both optical and auditory feedback to the surgeon after the injection of protoporphyrin IX. Patients in this series all had optical output guided resection. Three patients had additional auditory output which facilitated additional resection. For patients with a resectable tumor, surgical resection using intraoperative image guidance (whether with CT, MRI or using PTIX) was safer. Perhaps more complete resections can be performed. This innovative additional strategy seems to be an easy and effective technology which positively impacts outcomes. I believe that this technique has significant potential benefit, and will likely be more widely applied at centers of excellence that are heavily involved in improving outcomes of glioma patients.

\section{Reference}

1) Shapiro WR: Multimodality therapy of malignant glioma. BNI Quarterly: 48-52, 1985

L. Dade LunsFord, M.D., F.A.C.S. Lars Leksell Professor of Neurological Surgery University of Pittsburgh Medical Center Pittsburgh, Pennsylvania, U.S.A. 